\title{
The Effect of Memantine on Functional Recovery of the Sciatic Nerve Crush Injury in Rats
}

\author{
Mohammad-Bagher GHAYOUR, Arash ABDOLMALEKI, Morteza BEHNAM-RASSOULI \\ Ferdowsi University of Mashhad, Faculty of Science, Department of Biology, Mashhad, Iran
}

\section{ABSTRACT}

\begin{abstract}
AIM: Following severe peripheral nerve injury (PNI), regeneration is often insufficient and functional recovery is incomplete. In this regard, glutamate $\mathrm{N}$-methyl-D-aspartate (NMDA) receptor antagonist such as Memantine have been shown to have neuroprotective effects. We evaluated the effects of Memantine against sciatic nerve crush injury in male Wistar Rats.
\end{abstract}

MATERIAL and METHODS: Memantine or vehicle was given parenteraly to rats for 7 days postoperative. In Memantine treatment groups, a single dose of agent ( 5 and $10 \mathrm{mg} / \mathrm{kg}$ ) was administered daily. The control group was given vehicle in the same manner. The rats were subjected to crush injury in the left sciatic nerve with non-serrated clamp for 30 seconds. Behavioural, electrophysiological and morphological alterations were evaluated during the experimental period.

RESULTS: Results showed that Memantine has no significant effect on regeneration process rate and functional recovery quality. In the sciatic functional index (SFI) test no significant difference was observed between Memantine treatment groups (5 and $10 \mathrm{mg} /$ $\mathrm{kg})$ at any week.

CONCLUSION: Since the major neuroprotective effect of Memantine is due to its protective activity against NMDA receptormediated excitotoxicity, it seems that glutamate excitotoxicity is less important in motor impairment due to sciatic nerve crush injury. It is clear that more research is needed to confirm these findings.

KEYWORDS: Memantine, Sciatic nerve, Motor function, Injury, Regeneration, Peripheral nerve injury, Motoneuron

\section{INTRODUCTION}

$\mathrm{P}$ eripheral nerves injuries result in loss of motor, sensory and autonomic function of the affected nerves. Spinal neural circuits undergo continuous functional and structural changes in response to a peripheral nerve injury (2). Some recent studies show that glutamate receptors may be involved in sensory and motor neuron apoptosis following severe nerve injury in rats $(14,17,25,50)$. Glutamate is an important excitatory neurotransmitter in the nervous system. Neural trauma triggers massive release of glutamate from injured cells (2). Overactivation of the N-methyl-Daspartate (NMDA) glutamate receptor subtype can result in a process called excitotoxicity that leads to cell death $(20,32)$. Excitotoxicity is due to excessive calcium ion influx which, in turn, triggers free radical formation and multiple pathways leading to the initiation of apoptotic-like damage $(1,5)$. Therefore, glutamate excitotoxicity is an attractive therapeutic target for attenuation of neural tissue damage $(13,32)$. Hence, NMDA receptor antagonists could potentially provide neuroprotective effects in several neurodegenerative diseases manifesting excessive stimulation of NMDA receptors, including stroke, nerve injury and neuropathic pain syndromes $(2,21)$. However, attempts to use potent competitive NMDA receptor antagonists as neuroprotectants have shown serious side effects in patients (42).

Memantine is a non-competitive $N$-methyl-d-aspartate glutamate (NMDA) receptor antagonist and is capable of blocking excitotoxicity (12). This drug only reduces excessive NMDA receptor activation when it is pathologically activated for long 
periods of time under excessive glutamate exposure (12). Many in vitro and in vivo studies have shown the neuroprotective effects of NMDA receptor antagonists. In vitro studies using neuronal cell cultures showed that glutamate excitotoxicity was inhibited by Memantine (46). Also, some studies showed that Memantine attenuated neuronal damage caused by spinal cord and optic nerve ischemia $(11,18)$. Memantine could limit neuronal loss in animal models of stroke and peripheral ganglion injury models $(7,43)$. In addition, Memantine has shown neuroprotective effects against amyotrophic lateral sclerosis (ALS) and multiple sclerosis (MS) disease models (47). Furthermore, several clinical trials also showed the beneficial effects of Memantine for the treatment of Alzheimer's disease (AD) (31) and Parkinson's disease (PD) (24). Therefore, due to the lack of sufficient information concerning the neuroprotective properties of Memantine on the functional recovery of sciatic nerve injury, this experimental study was conducted to evaluate the probable effect of Memantine on functional recovery of crushed sciatic nerves in Wistar rats.

\section{MATERIAL and METHODS}

All animal experiments were carried out in accordance with the European Communities Council directive of 24 November 1986 (86/609/EEC), and in accordance with the local Ferdowsi University of Mashhad (FUM) committee for Human and Animal ethics.

Memantine and dimethyl sulfoxide (DMSO) were purchased from Sigma (USA) while ketamine and xylazine were obtained from Alfasan Pharmaceutical Co. (Holland) in injectable form. Memantine was dissolved in DMSO. All drugs were injected intraperitoneally (i.p.) and fresh drug solutions were prepared each day of the experiments.

All experiments were performed on adult male Wistar rats (weighing 250-300 g, aged 3 months). Animals were maintained under standardized housing conditions (temperature, $22 \pm 2^{\circ} \mathrm{C}, 12-\mathrm{h}$ light/dark cycle light on from 7 a.m. and $60 \pm 5 \%$ humidity) in plexiglas cages with free access to food (standard laboratory rodent chow) and tap water ad libitum. Experiments were carried out between 9 a.m. and 12 p.m. Ten rats were used for each treatment group.

All experiments were performed under an operating microscope in sterile conditions by the same investigator. All animals were deeply anesthetized using an intraperitoneal injection of ketamine $(80 \mathrm{mg} / \mathrm{kg})$ and xylazine $(10 \mathrm{mg} / \mathrm{kg})$. The skin was shaved and disinfected using $10 \%$ povidone iodine. Then, the rats were fixed in the prone position on the operating table under sterile conditions. The left sciatic nerve was exposed through a longitudinal incision extending from the greater trochanter to the mid-thigh. Then, a $3 \mathrm{~mm}$-long segment of the sciatic nerve was crushed by maximally clamping the nerve with non-serrated hemostatic forceps (Belge de Gembloux, Belgium) for $30 \mathrm{~s}$ at a position $1 \mathrm{~cm}$ below the sciatic notch. This procedure causes axonal interruption but preserves the connective sheaths (axonotmesis). For limiting the inter-animal variability in the postoperative outcome followed by microsurgical neurorrhaphy, the crush model was used. The crush model is appropriate to assess the roles of different agents in the nerve regeneration process and for pharmacological investigation (41). All surgeries were done using the same forceps. The nerves were kept moist with $37^{\circ} \mathrm{C}$ sterile saline solution throughout the surgical intervention. The crush site was marked with a 10-0 nylon suture (Alcon). In the sham-operated group, the left sciatic nerve was treated in the same way except for the crush. Finally, the muscle and skin were sutured with 6-0 nylon and rats were allowed to recover spontaneously from anesthesia. After recovery, rats were housed in groups of three per cage and received buprenorphine $(1 \mathrm{mg} / \mathrm{kg})$ for three days after surgery. To prevent autotomy, bitter nail polish was applied to each rat's left foot. During the study, animals were examined for signs of autotomy and contracture.

Fifty rats with sciatic nerve crush were randomly allocated into five groups $(n=10)$. In the two experimental groups, the animals were treated daily with Memantine at the doses of 5 or $10 \mathrm{mg} / \mathrm{kg}$ within 7 days after surgery. These selected doses of Memantine had a neuroprotective effect in experimental autoimmune encephalomyelitis (28). Controls were injected with vehicle (DMSO) and the sham-operated group was subjected to the surgical procedure without the nerve crush.

The recovery of motor function was assessed by calculating the sciatic functional index (SFI) at 1, 3, 5, 7 and 9 weeks after crush injury.

SFI test was performed in a confined corridor $(100 \times 10 \times 20$ $\mathrm{cm}$ ) with a dark box at the end. A white paper was placed on the floor of the corridor. Before the surgery, all rats were trained to walk in the corridor. Once the animals had learned to walk along the runway without stopping, their footprints were recorded. To record the footprints, the hind paws of rats were pressed down onto a finger paint-soaked sponge. Then animals were allowed to walk down the corridor leaving their hind footprints on the paper. The SFI value was calculated by putting the obtained data in the formula: SFI $=-38.3[(\mathrm{EPL}-$ NPL)/NPL] + 109.5[(ETS-NTS)/NTS] + $13.3[($ EIT-NIT)/NIT] 8.8, where EPL: the experimental paw length, NPL: the normal paw length, ETS: the experimental toe spread, NTS: the normal toe spread, EIT: the experimental intermediary toe spread and NIT: the normal intermediary toe spread (3). The SFI value varies from 0 to -100 , with 0 corresponding to normal function and -100 indicating total impairment. When no footprints were measurable, the index score of -100 was given (10). In each walking track, three footprints were analyzed by a single observer and the average of the measurements was used in SFI calculations.

At 5 and 9 weeks postoperative, non-invasive compound muscle action potential (CMAP) recording was performed in all animals following anaesthesia by intraperitoneal injection of ketamine $(80 \mathrm{mg} / \mathrm{kg})$ and xylazine $(10 \mathrm{mg} / \mathrm{kg})$. CMAPs were recorded in the gastrocnemius muscle by surface stimulation via the tendon-belly method (27), using an electrophysiological apparatus (CEPTU, England) with the PicoScope System Software. The sciatic nerve was stimulated by bipolar stimulating electrodes, which were placed on the skin premoistened with gel electrode, just between the 
ischial tuberosity and major trochanter and parallel to the sciatic nerve. The active and reference monopolar needle electrodes were inserted into the mid-belly and muscle tendon surface, respectively. A ground electrode was clamped to the skin, between the stimulating and recording electrodes. Stimulations with durations of $0.02 \mathrm{~ms}$ were administered at gradually increasing intensity until a maximal CMAP response was obtained. The recording was repeated three times, and the amplitude and latency of CMAP were averaged for each rat. Normal CMAPs were measured from the contralateral uninjured sides. All acquired data were entered into the computer to calculate the electrophysiological parameters of the regenerated nerve.

At 9 weeks postoperative, following the electrophysiology study, the animals were deeply anaesthetized with an intraperitoneal injection of ketamine and xylazine cocktail, and the distal parts of the crushed site of the left sciatic nerves were harvested from each group. Nerve samples were fixed in $4 \%$ paraformaldehyde and post-fixed in $1 \%$ osmium tetroxide. After dehydration with ascending ethanol passages, the specimens were embedded and serial crosssections of the distal zone for each nerve were cut. Cross sections of nerves were conducted starting $1 \mathrm{~mm}$ distally to the distal nylon suture (where the distal stump had been originally sutured) in order to allow visualization of regenerated fibers entering the distal nerve stump. For histomorphometric analysis, paraffin sections were stained with $1 \%$ toluidine blue (29). Axon counts, axon diameter and myelin thickness were calculated using the Image J program. The contralateral sciatic nerves were used as controls.

Muscle weight was measured to assess denervation atrophy at 9 weeks postoperative and after the electrophysiological test. The gastrocnemius muscles were harvested from both the experimental and contralateral (control) sides in each group. After bloodstain removal, the muscle was weighed while still wet using a digital scale (Sartorius, Germany). Then the gastrocnemius muscle mass ratio of the operated side to the contralateral side was calculated (muscle mass ratio $=$ weight of experimental muscle/weight of contralateral muscle). The index percentage produced represented the recovery in denervation atrophy of the gastrocnemius muscle on the operated side, with approximately $100 \%$ gastrocnemius muscle index (GMI) indicating full recovery of the operated side (19).

Data were analysed with SPSS Statistics 16.0 software (SPSS Inc., Chicago, Illinois, USA). Statistical analysis was carried out using a one-way analysis of variance (ANOVA) to determine the significant differences between the five groups. Intergroup comparison of means was performed using a Tukey-Post hoc analysis. All data were expressed as mean \pm standard error of mean (SEM) and values of $P<0.05$ were considered statistically significant.

\section{RESULTS}

Immediately after crushing the sciatic nerves (Figure 1), the compression areas were flattened but epineurium sheath continuity was preserved. All animals developed flaccid paralysis of the operated foot and survived with no wound infection.

\section{Functional Evaluation}

According to statistical data, the mean SFI value in the shamoperated group was approximately $(8.12 \pm 0.79)$ throughout the study, indicating normal function. One week after sciatic nerve crush, the SFI values in all groups decreased dramatically to the lowest level (near to -100 ) with a significant difference compared to sham-operated animals, indicating complete loss of function $(p<0.05$; Figure 2). Afterwards, all groups showed a time-dependent increase in SFI value with no significant differences between Memantine treatment and control groups in any week post-injury (Figure 2). Also, no significant difference was observed between Memantine treatment groups (5 and $10 \mathrm{mg} / \mathrm{kg}$ ) at any week (Figure 2). At the $9^{\text {th }}$ week, the SFI level of the Memantine treatment and

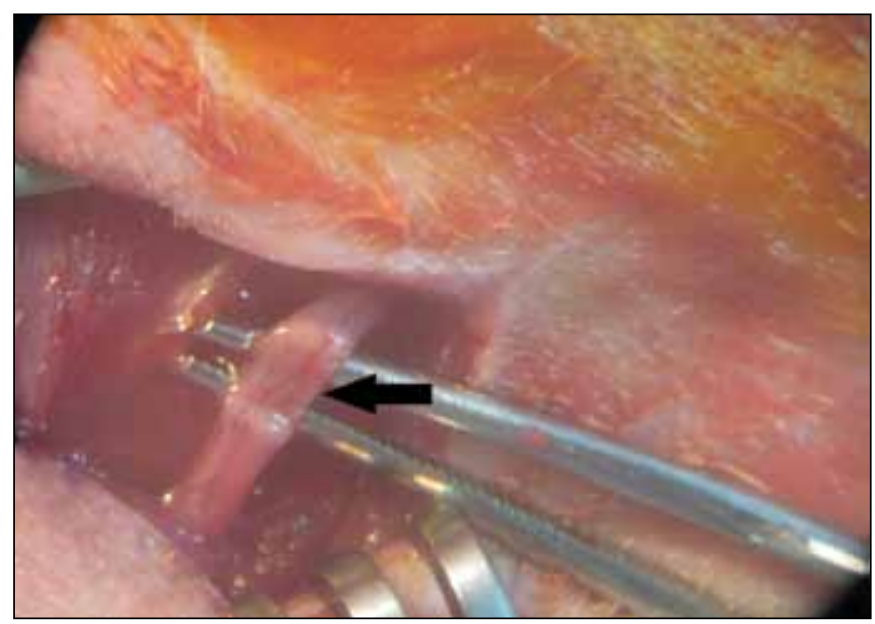

Figure 1: Picture showing the site of the sciatic nerve crush (arrow).

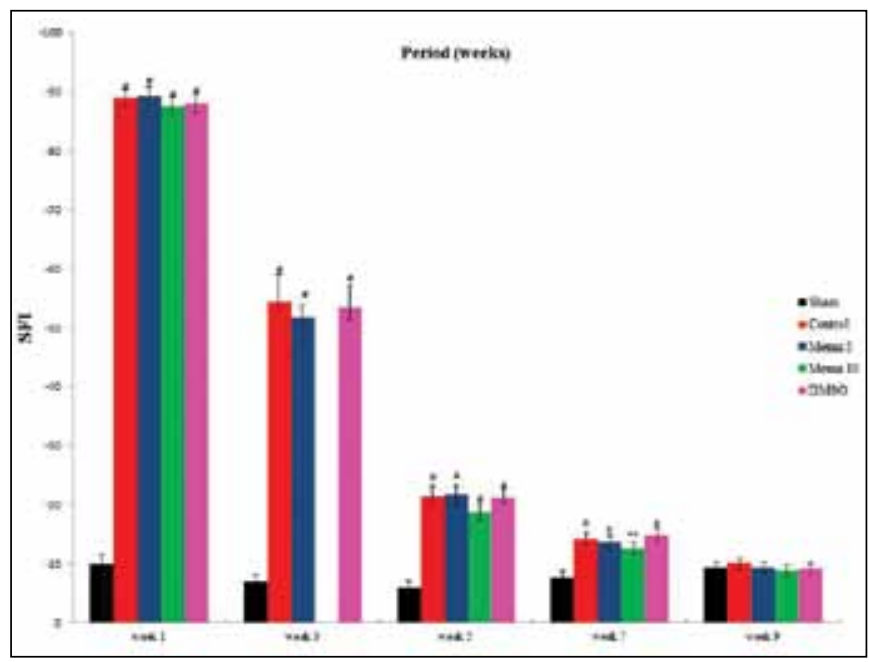

Figure 2: Sciatic function index (SFI) measured every other week after injury in each experimental group. Data were expressed as mean \pm SEM $(n=10)$. ${ }^{*} p<0.001$ and ${ }^{*} p<0.05$ vs. the sham group at the same time point. 
control groups were not significantly different compared with the sham-operated group and SFI returned to the baseline values of the preoperative period (Figure 2). These results indicate that Memantine treatment did not affect recovery of sciatic nerve motor function.

\section{Electrophysiological Evaluation}

To assess the regeneration process, electrophysiological recordings were conducted at the 5 and 9 weeks endpoints. For this purpose, the CMAP peak amplitudes and CMAP onset latencies were measured. In all groups, the CMAP amplitudes increased and CMAP onset latencies decreased progressively with time (Figure 3). At 5 weeks post-injury, CMAP amplitude values showed a significant difference between Memantine treatment and control groups compared with the shamoperated control group $(p<0.05)$. However, no significant difference was observed between Memantine treatment and control groups (Figure 3). At the end of 9 weeks, there was no significant difference between the CMAP amplitude in any group (Figure 3). There was also no significant difference between the Memantine treatment and control groups in terms of CMAP onset latencies at the same time (Figure 4). However, CMAP onset latencies in the sham-operated group were significantly shorter than that recorded from the Memantine treatment and control groups $(p<0.05$; Figure 4$)$.

\section{Histomorphometric Analysis}

According to quantitative morphometric analyses of the regenerated nerves, regenerating myelinated axons in the distal to injury site were found to be densely populated, with thinner myelin in comparison with the sham-operated group, at 9 weeks after crush $(p<0.05$; Table I). Increase in nerve fiber counts at the distal segment of the regenerated nerve may be due to axon-sprouting into the distal nerve (22). However, morphometric parameters such as the mean axonal

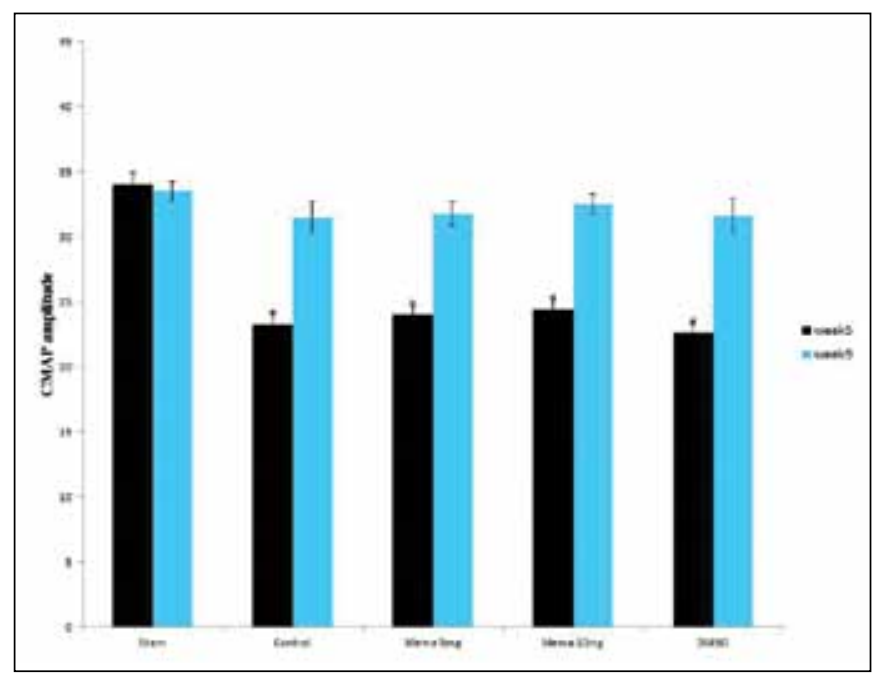

Figure 3: Representative results of CMAP amplitude measurements after proximal stimulation of operated and unoperated sciatic nerve at the $5^{\text {th }}$ and $9^{\text {th }}$ weeks post-injury. The data are shown as mean \pm SEM $(n=10)$. ${ }^{*} p<0.001$ and ${ }^{*} p<0.05$ vs. the sham group. number and myelin thickness showed no significant difference between the Memantine treatment and control groups (Table l).

\section{Muscle Mass}

At post-operative week 9 , the mean ratios of gastrocnemius muscles weight were measured. The gastrocnemius muscles in the operated limbs exhibited atrophy compared with the contralateral side in all groups. The results showed that there was no significant difference in the severity of muscle atrophy between the Memantine treatment and control groups. Therefore, Memantine treatment could not reduce muscle atrophy (Figure 5).

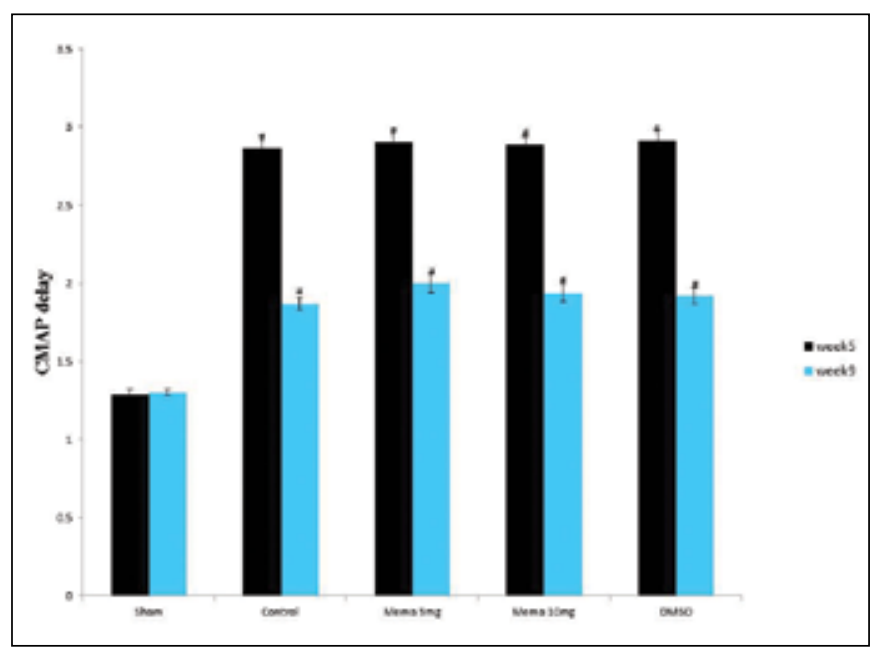

Figure 4: Representative results of CMAP delay measurements at the $5^{\text {th }}$ and $9^{\text {th }}$ weeks post-injury. The data are shown as mean \pm SEM $(n=10) .{ }^{*} p<0.001$ and ${ }^{*} p<0.05$ vs. the sham group.

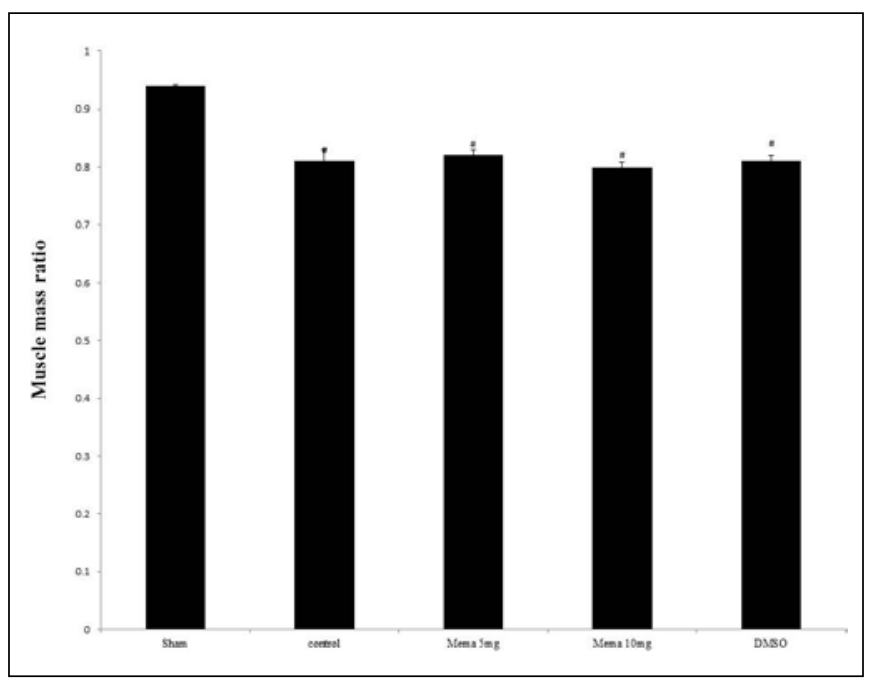

Figure 5: Gastrocnemius muscle mass ratio measurement. The gastrocnemius muscles of operated and unoperated sides were excised and weighed in the experimental groups at 9 weeks postoperatively. The data are shown as mean \pm SEM $(n=10)$. $p<0.001$ and ${ }^{*} \mathrm{p}<0.05$ vs. the sham group. 
Ghayour MB. et al: Effect of Memantine on Sciatic Nerve Injury

Table I: Morphometric Analyses of Transverse Sections at the Sciatic Nerve Distal to Injury for Each of the Experimental Groups 9 Weeks Post-Injury

\begin{tabular}{lcccc}
\hline Groups & Number of fibers & Diameter of fibers & Diameter of axon & Thickness of myelin sheath \\
\hline Sham & $7594.75 \pm 92.64$ & $6.29 \pm 0.08$ & $3.73 \pm 0.06$ & $1.28 \pm 0.02$ \\
\hline Control & $9567.12 \pm 105.41$ & $4.35 \pm 0.07$ & $2.84 \pm 0.07$ & $0.76 \pm 0.01$ \\
\hline Memantine $5 \mathrm{mg} / \mathrm{kg}$ & $9605.75 \pm 90.61$ & $4.27 \pm 0.08$ & $2.79 \pm 0.05$ & $0.74 \pm 0.02$ \\
\hline Memantine $10 \mathrm{mg} / \mathrm{kg}$ & $9703.75 \pm 107.65$ & $4.34 \pm 0.11$ & $2.86 \pm 0.06$ & $0.74 \pm 0.03$ \\
\hline DMSO & $9584.25 \pm 82.18$ & $4.3 \pm 0.08$ & $2.8 \pm 0.07$ & $0.75 \pm 0.02$ \\
\hline
\end{tabular}

Values are Shown as mean $\pm S D$. ${ }^{\#} P<0.001$ and ${ }^{*} P<0.05$ vs. sham group.

\section{DISCUSSION}

Peripheral nerve injuries make up $10 \%$ of all injuries and $30 \%$ of extremity injuries (33). Reinnervation time depends on the level of the lesion and whether the nerve division is far from the target organs. After intermediate-and low-level repairs, recovery was more useful than high-level repairs (33). The nature of the peripheral nerve demands a complex reparative process after nerve division. The goal of nerve repair is to bring the proximal and distal ends of the nerve, the fascicles or the fascicle groups together into close apposition without tension (8). Following nerve trauma, administration of neuroprotective agents is an appropriate strategy to control damage and promoting nerve regeneration process (38). Memantine is a non-competitive antagonist of the NMDA receptor with neuroprotective effect against some neurological disorders (16). In vitro studies using neuronal cell cultures showed that neuronal damage induced by glutamate was inhibited by Memantine (46). In vivo studies indicated that Memantine prevents the progression of neuronal loss in the amyotrophic lateral sclerosis and multiple sclerosis disease models (47). Furthermore, clinical trials showed the beneficial effects of Memantine for the treatment of $A D$ (31) and PD (24). Some studies showed that Memantine might prevent the development of central sensitization and neuropathic pain (26, 37). Also, a neuroprotective effect of Memantine against rabbit model of optic nerve ischemia (18), rat model of retinal injury (48) and spinal cord injury (11) has been reported. The result of a recent study, conducted by Topdag et al. in 2015, suggested that Memantine improves functional recovery of the facial nerve after crush injury (40). On the other hands, our unpublished data showed that early administration of Riluzole, a sodium channel blocker and anti-glutamatergic agent, after sciatic nerve crush could delay motor function recovery despite its well documented neuroprotective effect in the central nervous system $(36,44)$. We propose that the inhibitory effect of Riluzole on the sodium current has a dominant role in the sciatic nerve crush model rather than its antiglutamatergic effect. Based on such findings, the present study was conducted to investigate the neuroprotective effect of Memantine on sciatic nerve crush injury in rat. Our results showed that daily parenteral administration of Memantine (5 and $10 \mathrm{mg} /$ $\mathrm{kg}$ ) immediately after sciatic nerve crush has no significant effect on nerve regeneration process rate and functional recovery quality. Memantine, a non-competitive antagonist of the NMDA receptor, reduces pathological overactivity of NMDA receptors while allowing physiological activation $(16,21)$. Peripheral nerve injury results in excess release of glutamate from primary sensory neurons into the dorsal horn of the spinal cord (15). Several recent studies have indicated that glutamate receptors may be involved in motoneuronal death following axotomy in neonatal rats. Motoneuronal death after axotomy in newborn rats can be augmented by NMDA application (23). Also, the dorsal horn content and basal release of the excitatory amino acids glutamate and aspartate are increased in rats that have experienced chronic constriction injury $(\mathrm{CCl})$ to a sciatic nerve (35). Glutamate is the major transmitter of primary sensory afferents including nociceptors (4) and of excitatory interneurons in the dorsal horn of the spinal cord (39). Excessive glutamate release in dorsal horn of spinal cord results in overactivation of NMDA receptors and central sensitization (49). NMDA antagonists such as Memantine resulted in a decrease in mechanical hyperalgesia and mechanical allodynia in neuropathic rats $(6,34)$. Since glutamate excitotoxicity is a major cause of neuronal death after ischemia and traumatic injury in the CNS (45), glutamate antagonists can reduce neural tissue damage via inhibiting glutamate excitotoxicity (30). However, according to our results, Memantine has no significant effect on regeneration process in the sciatic nerve crush model. The major neuroprotective effect of Memantine is due to its protective activity against NMDA receptor-mediated excitotoxicity (9). It seems that, glutamate excitotoxicity is less important in motor impairment due to sciatic nerve crush injury.

\section{CONCLUSION}

Our study showed that Memantine given after crush injury has no significant effect on motor function recovery in the sciatic nerve crush model in rat. However, neuroprotective effects of NMDA antagonists such as Memantine in traumatic injuries of CNS are well documented. These differences may be due to different neural degeneration process and recovery in CNS and PNS. It is clear that more research is needed to confirm these findings. However, peripheral nerve transection causes more severe damage and apoptosis due to glutamate excitotoxicity in neurons of the dorsal root ganglion and ventral horn of the spinal cord. We suggest studying the effect of Memantine on recovery from a sciatic nerve transection. 


\section{- REFERENCES}

1. Ankarcrona M, Dypbukt JM, Bonfoco E, Zhivotovsky B, Orrenius S, Lipton SA, Nicotera P: Glutamate-induced neuronal death: A succession of necrosis or apoptosis depending on mitochondrial function. Neuron 15(4): 961-973, 1995

2. Azkue JJ, Zimmermann M, Hsieh TF, Herdegen T: Peripheral nerve insult induces NMDA receptor-mediated, delayed degeneration in spinal neurons. Eur J Neurosci 10(6): 22042206, 1998

3. Bain J, Mackinnon S, Hunter D: Functional evaluation of complete sciatic, peroneal, and posterior tibial nerve lesions in the rat. Plast Reconstr Surg 83(1): 129-136, 1989

4. Basbaum Al, Bautista DM, Scherrer G, Julius D: Cellular and molecular mechanisms of pain. Cell 139(2): 267-284, 2009

5. Bonfoco E, Krainc D, Ankarcrona M, Nicotera P, Lipton SA: Apoptosis and necrosis: Two distinct events induced, respectively, by mild and intense insults with $\mathrm{N}$-methyl-Daspartate or nitric oxide/superoxide in cortical cell cultures. Proc Natl Acad Sci 92(16): 7162-7166, 1995

6. Carlton SM, Hargett GL: Treatment with the NMDA antagonist memantine attenuates nociceptive responses to mechanical stimulation in neuropathic rats. Neurosci Lett 198(2): 115-118, 1995

7. Chen H-S, Wang Y, Rayudu P, Edgecomb P, Neill J, Segal $M$, Lipton SA, Jensen FE: Neuroprotective concentrations of the N-methyl-D-aspartate open-channel blocker memantine are effective without cytoplasmic vacuolation following postischemic administration and do not block maze learning or long-term potentiation. J Neurosci 86(4): 1121-1132, 1998

8. Daneyemez M, Solmaz I, Izci Y: Prognostic factors for the surgical management of peripheral nerve lesions. Tohoku J Exp Med 205(3):269-275, 2005

9. Danysz W, Parsons CG: The NMDA receptor antagonist memantine as a symptomatological and neuroprotective treatment for Alzheimer's disease: Preclinical evidence. Int J Geriatr Psychiatry 18: S23-S32, 2003

10. Dijkstra JR, Meek MF, Robinson PH, Gramsbergen A: Methods to evaluate functional nerve recovery in adult rats: Walking track analysis, video analysis and the withdrawal reflex. $J$ Neurosci Methods 96(2):89-96, 2000

11. Ehrlich M, Knolle E, Ciovica R, Böck P, Turkof E, Grabenwöger M, Cartes-zumelzu F, Kocher A, Pockberger H, Fang C, Wolner E, Havel M: Memantine for prevention of spinal cord injury in a rabbit model. J Thorac Cardiovasc Surg 17(2): 285-291, 1999

12. El Nasr MS, Peruche B, Roßberg C, Mennel HD, Krieglstein $\mathrm{J}$ : Neuroprotective effect of memantine demonstrated in vivo and in vitro. Eur J Pharmacol 185(1):19-24, 1990

13. Epstein FH, Lipton SA, Rosenberg PA: Excitatory amino acids as a final common pathway for neurologic disorders. $\mathrm{N}$ Engl $\mathrm{J}$ Med 330(9): 613-622, 1994

14. Groves MJ, Christopherson T, Giometto B, Scaravilli F: Axotomy-induced apoptosis in adult rat primary sensory neurons. J Neurocytol 26(9): 615-624, 1997
15. Inquimbert P, Bartels K, Babaniyi OB, Barrett LB, Tegeder I, Scholz J: Peripheral nerve injury produces a sustained shift in the balance between glutamate release and uptake in the dorsal horn of the spinal cord. Pain 153(12): 2422-2431, 2012

16. Johnson JW, Kotermanski SE: Mechanism of action of memantine. Curr Opin Pharmacol 6(1): 61-67, 2006

17. Kashihara $Y$, Kuno M, Miyata Y: Cell death of axotomized motoneurones in neonatal rats, and its prevention by peripheral reinnervation. J Physiol 386(1): 135-148, 1987

18. Kim TW, Kim DM, Park KH, Kim H: Neuroprotective effect of memantine in a rabbit model of optic nerve ischemia. Korean J Ophthalmol 16(1):1-7, 2002

19. Lewin SL, Utley DS, Cheng ET, Verity AN, Terris DJ: Simultaneous treatment with BDNF and CNTF after peripheral nerve transection and repair enhances rate of functional recovery compared with BDNF treatment alone. Laryngoscope 107(7):992-999, 1997

20. Lipton SA, Nicotera P: Calcium, free radicals and excitotoxins in neuronal apoptosis. Cell Calcium 23(2):165-171, 1998

21. Lipton SA: Failures and successes of NMDA receptor antagonists: Molecular basis for the use of open-channel blockers like memantine in the treatment of acute and chronic neurologic insults. NeuroRx 1(1): 101-110, 2004

22. MacKinnon SE, Dellon A, O'Brien J: Changes in nerve fiber numbers distal to a nerve repair in the rat sciatic nerve model. Muscle nerve 14(11):1116-1122, 1991

23. Mentis GZ, Greensmith L, Vrbova G: Motoneurons destined to die are rescued by blocking $\mathrm{N}$-methyl-D-aspartate receptors by MK-801. Neuroscience 54(2):283-285, 1993

24. Merello M, Nouzeilles MI, Cammarota A, Leiguarda R: Effect of memantine (NMDA antagonist) on Parkinson's disease: A double-blind crossover randomized study. Clin Neuropharmacol 22(5): 273-276, 1999

25. Miyata $\mathrm{Y}$, Kashihara $\mathrm{Y}$, Homma S, Kuno M: Effects of nerve growth factor on the survival and synaptic function of la sensory neurons axotomized in neonatal rats. J Neurosci 6(7): 2012-2028, 1986

26. Morel V, Etienne M, Wattiez AS, Dupuis A, Privat AM, Chalus $M$, Eschalier A, Daulhac L, Pickering G: Memantine, a promising drug for the prevention of neuropathic pain in rat. Eur J Pharmacol 721(1): 382-390, 2013

27. Oguzhanoglu A, Erdogan C, Tabak E, Cenikli U: Comparison of conduction velocities of nerve fibers to smaller and larger muscles in rats. Int J Neurosci 120(1): 76-79, 2010

28. Paintlia AS, Paintlia MK, Singh I, Singh AK: Combined medication of lovastatin with rolipram suppresses severity of experimental autoimmune encephalomyelitis. Exp Neurol 214(2):168-180, 2008

29. Raimondo S, Fornaro M, Di Scipio F, Ronchi G, GiacobiniRobecchi MG, Geuna S: Methods and protocols in peripheral nerve regeneration experimental research: Part IImorphological techniques. Int Rev Neurobiol 87: 81-103, 2009

30. Rao VLR, Dogan A, Todd KG, Bowen KK, Dempsey RJ: Neuroprotection by memantine, a non-competitive NMDA receptor antagonist after traumatic brain injury in rats. Brain Res 911(1):96-100, 2001 
31. Reisberg B, Doody R, Stöffler A, Schmitt F, Ferris S, Möbius $\mathrm{HJ}$ : Memantine in moderate-to-severe Alzheimer's disease. $\mathrm{N}$ Engl J Med 348(14): 1333-1341, 2003

32. Rothman SM, Olney JW: Glutamate and the pathophysiology of hypoxic-ischemic brain damage. Ann Neurol 19(2):105111,1986

33. Secer HI, Daneyemez M, Tehli O, Gonul E, Izci Y: The clinical, electrophysiologic, and surgical characteristics of peripheral nerve injuries caused by gunshot wounds in adults: A 40-year experience. Surg Neurol 69(2):143-152, 2008

34. Smith G, Wiseman J, Harrison S, Elliott P, Birch P: Pre treatment with MK-801, a non-competitive NMDA antagonist, prevents development of mechanical hyperalgesia in a rat model of chronic neuropathy, but not in a model of chronic inflammation. Neurosci Lett 165(1):79-83, 1994

35. Somers DL, Clemente FR: Dorsal horn synaptosomal content of aspartate, glutamate, glycine and GABA are differentially altered following chronic constriction injury to the rat sciatic nerve. Neurosci Lett 323(3):171-174, 2002

36. Stutzmann JM, Pratt J, Boraud T, Gross C: The effect of riluzole on post-traumatic spinal cord injury in the rat. Neuroreport 7(2): 387-392, 1996

37. Suzuki R, Matthews EA, Dickenson AH: Comparison of the effects of MK-801, ketamine and memantine on responses of spinal dorsal horn neurones in a rat model of mononeuropathy. Pain 91(1): 101-109, 2001

38. Terenghi G, Hart A, Wiberg M: The nerve injury and the dying neurons: Diagnosis and prevention. J Hand Surg 36(9):730734, 2011

39. Todd AJ: Neuronal circuitry for pain processing in the dorsal horn. Nat Rev Neurosci 11(12):823-836, 2010

40. Topdag M, Topdag DO, Ila K, Muezzinoglu B, Yaprak B, Ozturk M, Caliskan S, Iseri M: The effect of memantine on functional recovery of the facial nerve after crush injury. Eur Arch Otorhinolaryngol 272(2): 473-478, 2015

41. Tos P, Ronchi G, Papalia I, Sallen V, Legagneux J, Geuna $S$, Giacobini-Robecchi M: Methods and protocols in peripheral nerve regeneration experimental research: Part IExperimental models. Int J Neurosci 87: 47-79, 2009
42. Volbracht C, Van Beek J, Zhu C, Blomgren K, Leist M: Neuroprotective properties of memantine in different in vitro and in vivo models of excitotoxicity. Eur J Neurosci 23(10): 2611-2622, 2006

43. Vorwerk CK, Lipton SA, Zurakowski D, Hyman BT, Sabel BA, Dreyer EB: Chronic low-dose glutamate is toxic to retinal ganglion cells. Toxicity blocked by memantine. Invest Ophthalmol Vis Sci 37(8): 1618-1624, 1996

44. Wahl F, Renou E, Mary V, Stutzmann JM: Riluzole reduces brain lesions and improves neurological function in rats after a traumatic brain injury. Brain Res 756(1): 247-255, 1997

45. Wang Q, Yu S, Simonyi A, Rottinghaus G, Sun GY, Sun AY: Resveratrol protects against neurotoxicity induced by kainic acid. Neurochem Res 29(11): 2105-2112, 2004

46. Weller M, Finiels-Marlier F, Paul SM: NMDA receptormediated glutamate toxicity of cultured cerebellar, cortical and mesencephalic neurons: Neuroprotective properties of amantadine and memantine. Brain Res 613(1): 143-148, 1993

47. Wenk GL, Parsons CG, Danysz W: Potential role of N-methyl$D$-aspartate receptors as executors of neurodegeneration resulting from diverse insults: Focus on memantine. Behav Pharm 17(5): 411-424, 2006

48. WoldeMussie E, Yoles E, Schwartz M, Ruiz G, Wheeler LA: Neuroprotective effect of memantine in different retinal injury models in rats. J Glaucoma 11(6): 474-480, 2002

49. Woolf CJ, Thompson SW: The induction and maintenance of central sensitization is dependent on N-methyl-D-aspartic acid receptor activation; implications for the treatment of post-injury pain hypersensitivity states. Pain 44(3):293-299, 1991

50. Zhou XF, Li WP, Zhou FH, Zhong JH, Mi JX, Wu LY, Xian CJ: Differential effects of endogenous brain-derived neurotrophic factor on the survival of axotomized sensory neurons in dorsal root ganglia: A possible role for the $\mathrm{p} 75$ neurotrophin receptor. J Neurosci 132(3): 591-603, 2005 